\title{
Discharge Characteristics in Soils Subjected to Lightning Impulse Voltages
}

\author{
Seung Min Kim*, Yang-Woo Yoo* and Bok-Hee Lee ${ }^{\dagger}$
}

\begin{abstract}
In this paper, we present experimental results of the soil discharge characteristics as a function of moisture content when a $1.2 / 50-\mu \mathrm{s}$ lightning impulse voltage is applied. For this study, laboratory experiments were carried out based on factors affecting the transient behavior in soils. The electrical breakdown voltages in soils were measured for a $0-6 \%$ range of moisture content for sand and a $0-4 \%$ range of moisture content for gravel. A test cell with semi-spherical electrodes buried face-to-face in the middle of a cylindrical container was used. The distance separating the electrodes is $100 \mathrm{~mm}$. As a result, the time-lag to breakdown in soils decreases as the amplitude of applied voltage increases. The time-lag to initiation of ionization streamer is decreased, with an increase in the moisture content. However, the formative time-lag is rarely changed. The behavior of soil discharges depend not only on the type of soil and its moisture content but also on the amplitude of the impulse voltage. When the test voltage is applied repeatedly, electrical breakdown occurs along different discrete paths, leading radially away from the injected electrode. i.e., the fact that the ionization streamers propagate in different paths from shot to shot was observed.
\end{abstract}

Keywords: Soil discharge, Lightning impulse voltage, Formative time-lag, Electrical breakdown, V-t curve

\section{Introduction}

It is very important to obtain some information regarding soil discharges when designing and preparing grounding systems for lightning protection. Lightning protection analysis requires the knowledge of the transient behavior of grounding systems. Voltages induced on buried conductors by a lightning strike can produce electrical breakdown in the surrounding earth [1]. Soil discharge has a noticeable influence on the ground potential rise from lightning strikes to air terminals. The transient performance of grounding systems is closely related to the behavior of grounding electrodes subjected to lightning return stroke currents. Also soil discharges are a critical factor to change in the transient performance of grounding systems, i.e., they may reduce the impulse impedance of grounding systems. Thus far, many researchers have realized extensive experiments in order to establish a model capable of describing the dynamic behavior of grounding electrode systems at high impulse voltages [2-5]. From many experimental and simulation results, it was concluded that the dynamic behavior of grounding systems at high impulse currents depends not only on the characteristics of soil ionization but also on the discharge mechanisms [6-11].

Some data observing the ground surface flashovers from the lightning strikes points were reported by some authors

$\dagger$ Corresponding Author: Dept. of Electrical Engineering, Inha University, Korea. (bhlee@inha.ac.kr)

* Dept. of Electrical Engineering, Graduate School, Inha University, Korea. (sm0822@inha.edu)

Received: March 28, 2015; Accepted: October 7, 2015
[12-14]. Fig. 1 shows an example of photographs of the ground surface flashover from the ground rod vertically driven at the test site with wet loamy sand [14].

When the ground impedance is not sufficient low, the flashover may be occurred on the ground surface touched with ground electrode stressed by a lightning strike. Perhaps the ground surface discharge can cause a danger of electric shock. If the electrical breakdown is quickly occurred at the ground electrode buried in soil, the safety for human and electrical installations may be ensured. In view of other necessary assumptions, this might be considered on the 'safe' side.

Consequently, electrical discharge in soils is an important research area that needs to be further investigated in order to understand better the transient response of grounding systems. Moreover, the physical parameters of

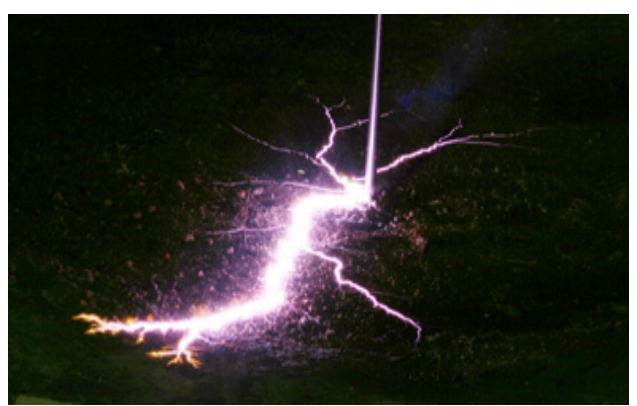

Fig. 1. Photograph of surface flashover from the driven ground rod impressed by a lightning impulse voltage. 
soil significantly affect the discharge characteristics caused by lightning impulse voltages. It is necessary to study soil discharge behavior depending on the grain size, composition, moisture content, resistivity, and permittivity, which are all related to the characteristics of the lightning impulse current passing through the soil. The knowledge of the exact electrical parameters of the soil is very useful. Indeed, it allows both material and cost savings while installing grounding systems for lightning protection. To the best of our knowledge, many studies on characteristics of transient impedance of grounding systems stressed by high impulse currents have been conducted $[15,16]$. However, data for soil discharges are still insufficient and required.

Understanding the phenomena of soil discharges is necessary to achieve high performance grounding systems capable of protecting the electrical and electronic equipment from lightning strikes. In this study, we conduct experimental analysis to analyze the impact of moisture content on the soil discharges near the grounding electrodes. The electrical breakdown voltage and the current passing through the soils stressed by the $1.2 / 50-\mu$ s lightning impulse voltage are measured in a hemispheric-shaped electrodes system. The time-lag to breakdown, the statistical time-lag, and the formative time-lag are evaluated as a function of the moisture content by weight in soil. The development processes of soil discharges are analyzed based on the current waveforms. Finally, the paths for electrical discharges, for repeatedly applied lightning impulse voltages, are examined and discussed.

\section{Experimental Procedure}

\subsection{Experimental setup}

Fig. 2 shows a schematic illustration of the experimental and measuring arrangements that consist of a Marx generator, a test chamber loading the test soil between the hemispherical electrodes, the voltage divider, the current monitor and the waveform recording system. Impulse voltage test system of $20 \mathrm{~kJ}$ and $400 \mathrm{kV}$, which is composed of an impulse voltage generator(Marx generator), measuring divider, charging voltage generator, data acquisition equipment, shorting and earthing device, was employed. The $1.2 / 50-\mu$ s lightning impulse voltage is

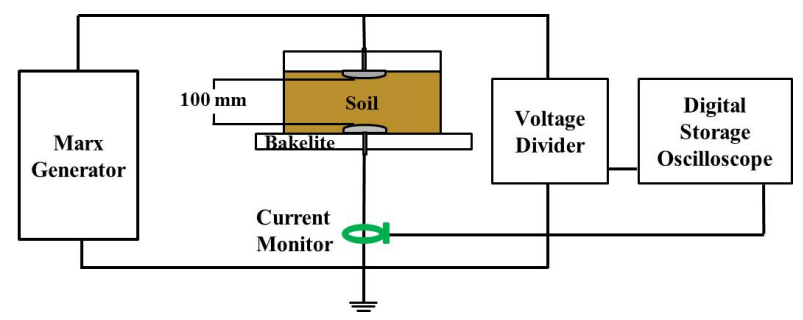

Fig. 2. Schematic diagram of the experimental setup produced by a Marx generator, with a peak voltage of 400 $\mathrm{kV}$ applied to the top electrode in the test container. The $1 \mu \mathrm{F}$ charging capacitors having the withstand voltage of $100 \mathrm{kV}$ were connected with four-stages in series.

In this study, the acryl cylinder container of $300 \mathrm{~mm}$ in diameter is adopted as a test container filled with the test soil. The test container was separated from the experimental bench by a bakelite plate of $10 \mathrm{~mm}$ in thickness. The hemispherical stainless steel electrodes of $75 \mathrm{~mm}$ in diameter are placed in the middle of the cylindrical container, at opposite sides. The spacing distance between the electrodes is $100 \mathrm{~mm}$.

A capacitive voltage divider with a ratio of $10,000: 1$ was used for the measurement of the voltage between the top and bottom electrodes. The output of the voltage divider is connected to an oscilloscope with coaxial cable having the 50-ohm characteristic impedance. The current passing through the test soil was measured using a current monitor whose sensitivity is $0.1 \mathrm{~V} / \mathrm{A}$. The voltage and current signals are displayed on a 4-channel $500 \mathrm{MHz}$ digital storage oscilloscope and are recorded in a digital format at a data acquisition system. The control devices and instruments are placed in a Faraday cage. Moreover, power line filters and an isolation transformer protect the oscilloscope's power supply from noise and disturbances.

\subsection{Experimental method}

The fine sand and gravel were adopted as the test samples because they are easily wetted and dried without change of physical properties. The size of a sand grain is less than $0.3 \mathrm{~mm}$ in diameter and the size of a gravel grain is in the range of $4-5 \mathrm{~mm}$ in diameter. The test soils are classified using a standard sieve.

In order to analyze the development characteristics of soil discharges as a function of moisture content, the moisture content of sand is controlled by adding the tap water with a resistivity of $70 \Omega \cdot \mathrm{m}$. The moisture content refers to the water content percentage measured by weight. The moisture contents of the test samples are in the 0-6\% range for fine sand and in the $0-4 \%$ range for gravel. Water is equally spread into the entire quantity of the test soils. The test soils are dried prior to sequential experiments in order to ensure uniform conditions for both sample soils. After loading the soil into the test container, it is compressed, by applying a uniform pressure of $5 \mathrm{kPa}$, to eliminate all remaining air gap between the soil grains.

When the applied voltage is lower than $40 \mathrm{kV}$, the electrical breakdown is not occurred in all our experimental conditions. However, when the applied voltage is higher than $260 \mathrm{kV}$, the test soil particles scatter during a shot. Thus the range of test voltage was limited and measurements were made of the time dependence of breakdown voltage between 40 to $260 \mathrm{kV}$. The breakdown voltage and the current passing through the test soil were measured versus the amplitude of applied voltage for sample soils and 
several moisture contents. The experiments observing soil discharges are performed as a function of moisture content. After changed moisture contents, experiments were carried out quickly so as to reduce an error due to evaporation of water. The temperature in the laboratory was about $26^{\circ} \mathrm{C}$ and the relative humidity was $48 \%$. The physical and electrical phenomena related to the effect of the soil discharge processes are analyzed and discussed based on the voltage and current waveforms, the electrical breakdown voltage, and the breakdown voltage-time (V-t) curves.

\section{Results and Discussion}

\subsection{Characteristics of soil discharge developments}

The main factors affecting the development process of electrical discharges in soils are the moisture content and grain size of soils. The electrical breakdown voltages and discharge currents in sand and gravel samples are measured as a function of the moisture content. After the change of moisture content, experiments were carried out rapidly in order to reduce measurement errors due to moisture vaporization. When the applied voltage is significantly high, the electrical breakdown is occurred in the test soils. The development processes of soil discharges can be inferred crudely from the breakdown voltage and current records.

Fig. 3 shows a typical set of time traces of the measured discharge current, the voltage across the test electrodes, and the calculated transient impedance for sand with $3 \%$ of moisture content. The high current soil discharges help to reduce the ground potential rise. The transient impedance is equal to the ratio between the instantaneous values of the developed voltage across the test soil and the discharge current.

When electrical breakdown by ionization begins, the voltage decreases rapidly while the current first increases and then decreases. After electrical breakdown, the transient impedance of the soil drastically decreases with time until

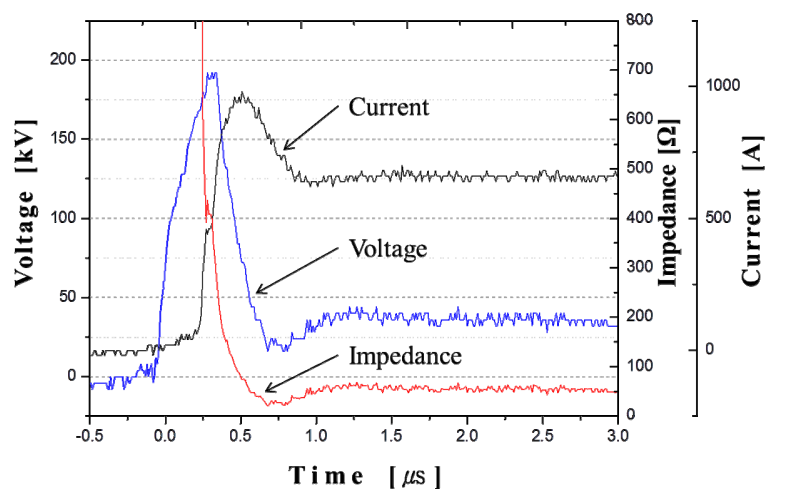

Fig. 3. Typical time traces of the discharge current, the observed voltage, and the transient impedance it reaches a minimum value.

The discharge currents are spread into the soils. The discharge expands radially away from the top electrode. The electrical discharges in soil form a number of branch channels that proceed away from the top electrodes. The electrical breakdown is noticed by the rapid increase of current and corresponding dropping of the voltage. In other words, a significant decrease of soil impedance occurs during the breakdown.

After breakdown, the current decreases slowly due to its characterization by the behavior of gap and line constants of the experimental setup (issued mainly from the Marx generator). The current duration of soil discharges is approximately $30 \mu \mathrm{s}$. The electrical breakdowns initiate voids in air. In the voids, the electric field is enhanced because of the discontinuity in the dielectric constant between the air and the soil. Consequently it is believed that the electrical breakdown in the sample soils is due to avalanche ionization in the air voids between the soil particles.

Fig. 4 shows a series of typical voltage and current traces for soil discharges, developed by a $1.2 / 50-\mu$ s lightning impulse voltage. The curve at the top of each sub-figure refers to the current behavior, whereas the curve at the bottom of each sub-figure is the voltage behavior. As can be seen, the current before the initiation of ionization is the prebreakdown leakage current through the test soil. Thereafter the current waveforms imply that the electrical breakdown propagates through two or more stages in small air pockets of the soil. The soil discharge is responsible for the reduction of the grounding electrode potential. The current flow mechanisms for electrical breakdowns are different for dry and wet soils.

Indeed, the current flow consists of two regimes as indicated in Fig. 4: conduction and ionization. That is, the discharge development processes are classified into two categories based on the current waveform: current behavior before ionization and current behavior after ionization. When the voltage level supplied between the test electrodes is relatively low, the conduction current runs into the test soil. The conduction current increases linearly with the amplitude of applied voltage. Furthermore, it has been noticed that the conduction current flowing through the test soils increases proportionally with the level of moisture content. When an impulse voltage, of sufficiently high value to cause electrical breakdown is applied, a spark bridges the air spaces between the dry soil grains. The conduction current prior to ionization in the dry soil is very low as shown in the current waveforms of Fig. 4. However, the conduction current in the wet soil is increased with the applied impulse voltage, because the wet soil grains act as conductors.

Fig. 5 illustrates the influence of the moisture content on the transient impedance, as a response to $1.2 / 50$ - $\mu \mathrm{S}$ lightning impulse voltage for analyzing the postbreakdown behavior. The transient impedance means the post- 


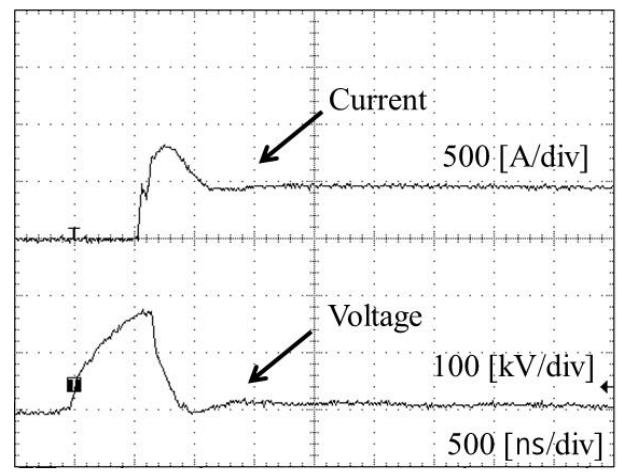

(a) Sand - moisture content: $0 \%$

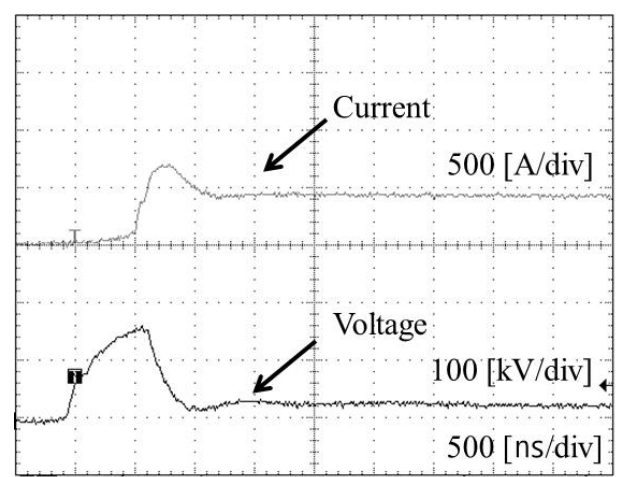

(b) Sand - moisture content: $6 \%$

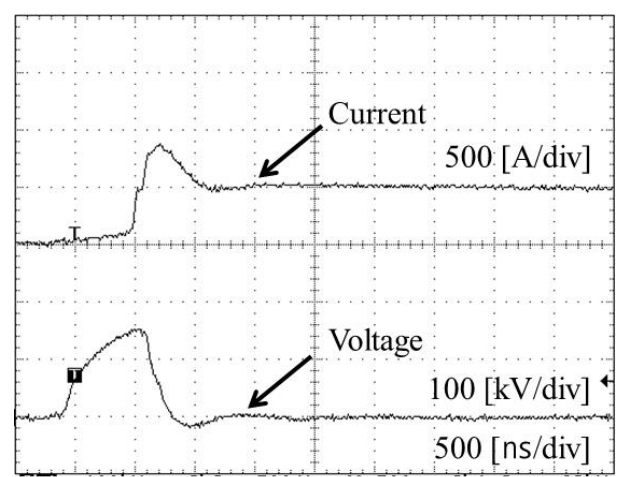

(c) Gravel - moisture content: $0 \%$

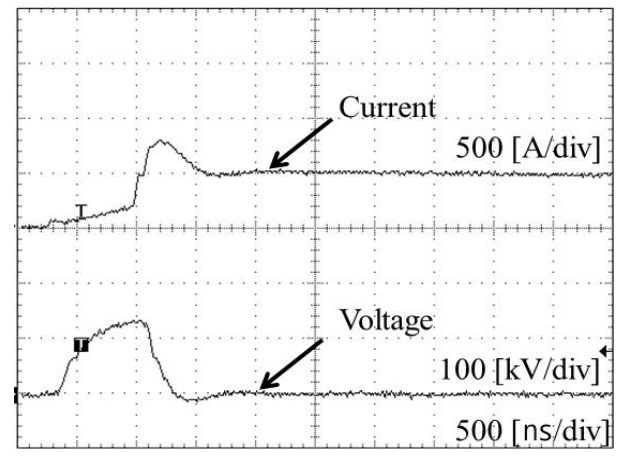

(d) Gravel - moisture content: 4\%

Fig. 4. Typical voltage and current waveforms for soil discharges caused by the $1.2 / 50-\mu$ s lightning impulse voltage. breakdown V-I characteristics of the soils, that is, the arc impedance [2]. When the applied impulse voltage is above the breakdown voltage value, the discharge current following the conduction current is abruptly increased and the discharge development comes to a final stage. When a sufficiently high voltage impulse is applied to a soil, a breakdown will occur at each impulse application. The nonlinear behaviors of voltage and current responses are caused by the discharge development. Hence, the ground impedance and the transient ground potential rise can be reduced on the surface of the ground. From the sub-figures $5(\mathrm{a})$ and $5(\mathrm{~b})$, it is noticed that the transient impedance curves shift towards early time for an increasing level of moisture content. The dependency of the transient impedance on the moisture content is more important for sand than for gravel.

Moreover, the impedance across the test soil is very high for conduction current and then is abruptly decreased at the initiation of ionization. The behavior of the transient impedance in time is similar for both types of soil. However, their values of impedance differ after breakdown. Indeed, in the case of sand, because the electrical breakdown occurs by ionization, the impedance drops after breakdown, and is then sustained at a very low value. However, because the electrical breakdown occurs by sparking through the air pockets between the gravel grains, the impedance drops to zero. Finally, the transient impedance curves present an

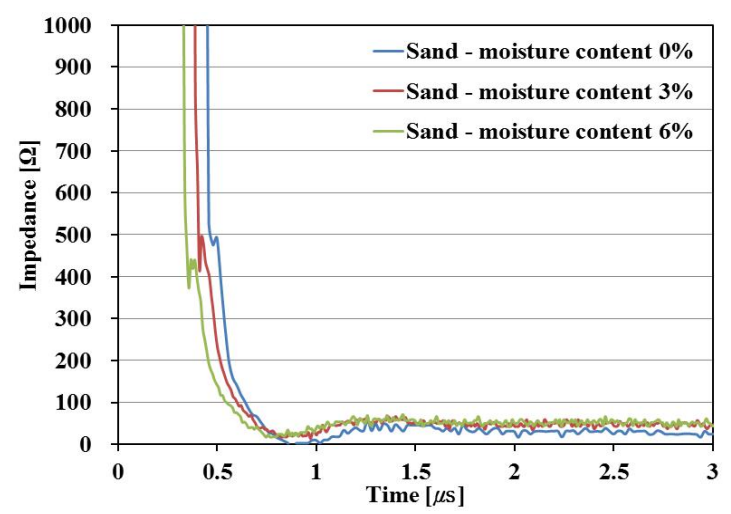

(a) Sand

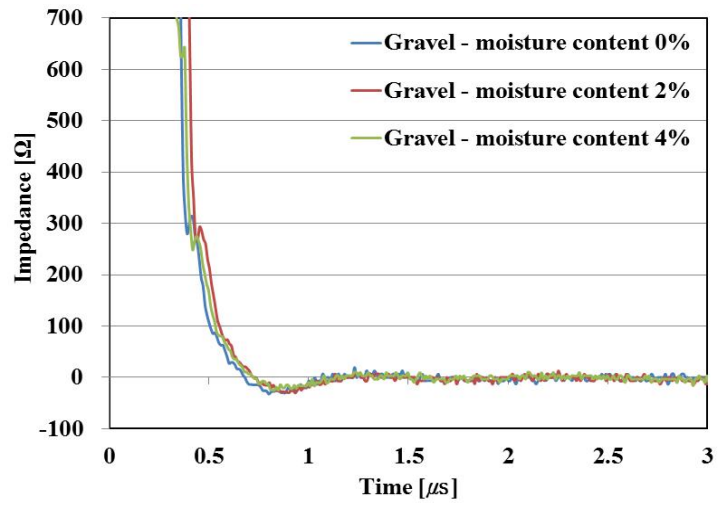

(b) Gravel

Fig. 5. Transient impedances for different types of soil 
under-damped oscillatory shape caused by the test circuit constants. Indeed, the tail parts depend on the capacitance of the Marx generator and on the inductance of connecting leads, because the resistance of the test soil at the sparking instant is practically zero.

\subsection{Breakdown voltage - Time-lag characteristics}

For the electrical breakdown, it is essential that the discharge occurs during the interval of overvoltage duration, i.e., the overvoltage duration must exceed the time-lag, that is the time elapsed between the application of an impulse voltage to a soil, sufficiently strong to cause breakdown, and the breakdown [17].

For the electrical breakdown under impulse voltages, there is a time-lag between the application of impulse voltage and the breakdown. This time-lag is believed to be the sum of a 'statistical time-lag' for the initiation of ionization streamer in the soil and a 'formative time-lag' for the streamer to propagate completely across the test soil, at which time the large breakdown current starts to flow [18]. Fig. 6 illustrates the effect of applied voltage impulse on the time-lag, in order to investigate the breakdown characteristics. The time to breakdown $\left(\mathrm{t}_{3}\right)$ consists of the statistical time-lag $\left(t_{1}\right)$ and the formative time-lag $\left(t_{2}\right)$. The statistical time-lag $t_{1}$ is the time between the application of a voltage sufficient to cause soil breakdown and the initiation of ionization, and it decreases as the amplitude of applied voltage increases.

Following two techniques are generally used for constructing a breakdown voltage - time curve: either a constant voltage is applied to a test gap or an overvoltage is suddenly applied to a test gap. In the former case, the timelag is measured from the flash until breakdown occurs: The measured time-lags for given experimental conditions are usually presented graphically by plotting the average timelags against the overvoltage. While in the latter the timelags under impulse voltages are measured between the voltage application and the breakdown, the time-lags are plotted against the amplitude impulse voltages. Our data for $\mathrm{V}$ - $\mathrm{t}$ characteristic curves were constructed by the latter technique.

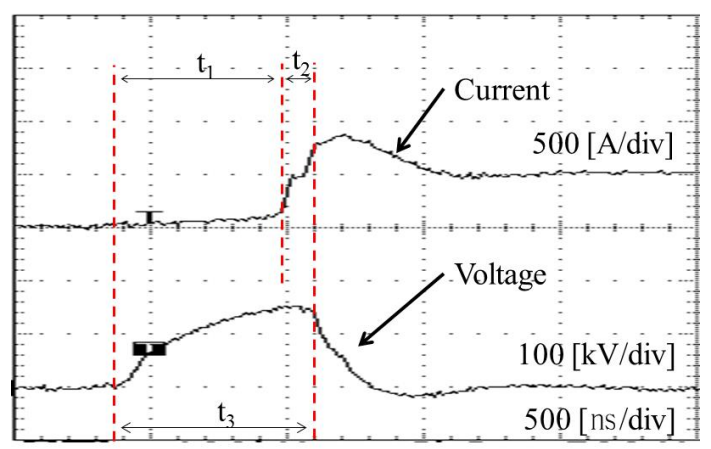

Fig. 6. Determination of the time required for the discharge development
The breakdown voltage - time (V-t) characteristics provide valuable information about the lightning impulse response of grounding systems. The time required for the discharge development (time-lag) depends on a number of factors, such as the rate of rise, the amplitude of impulse voltage, the gap geometry, the nature and the condition of the electrode surface, and the type and moisture content of soil.

The electrical breakdown voltage $(\mathrm{V})$ versus the time to breakdown $\left(\mathrm{t}_{3}\right)$ characteristics as a function of moisture content in soils are determined by the time interval from the origin to breakdown, and the polarity is designated by the polarity of voltage injected to the top electrode. The V-t curves are plotted by adopting the maximum voltage amplitude recorded at or prior to breakdown, which is the test procedures recommended in the international standard IEC 60060-1 [19]. Since water evaporation in soil takes place due to excessive discharge current, the repeated experiments can cause the irregularity of measured data at a specific condition of the test soil. Thus water evaporation in soil should minimized by limiting the number of impulse shots.

It is possible to construct a breakdown voltage-time characteristic curve by applying a number of impulse voltage of increasing the amplitude and noting oscillographically

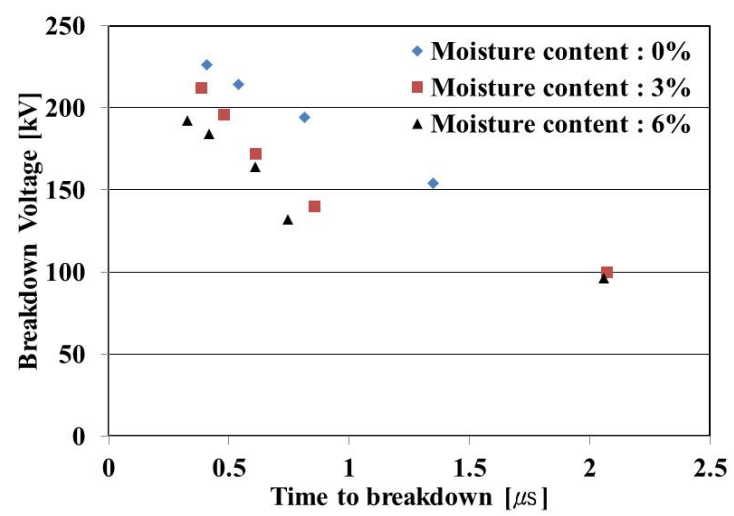

(a) Sand

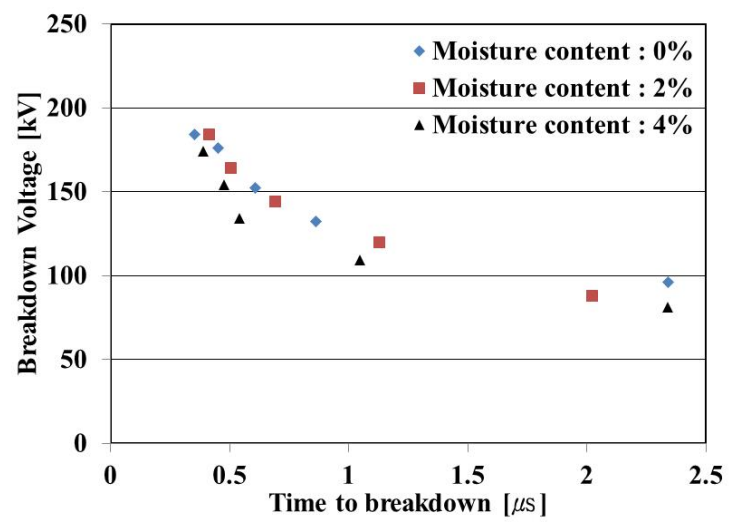

(b) Gravel

Fig. 7. Breakdown voltage - time $(\mathrm{V}-\mathrm{t})$ curves depending on the moisture content in soils 
the time-lag. The trend of electrical breakdown behavior of soils are similar to the observed characteristics in other researches $[18,20]$. Fig. 7 shows the breakdown voltagetime (V-t) curves for sand and gravel samples. i.e., the V-t curve illustrates a plot of the necessary time to occur the electrical breakdown as a function of moisture content.

The moisture content of soil is the critical parameter that affects the breakdown channel. The measured values are affected by factors such as types of soil, the moisture content and the amplitude of applied voltage. When soils have different moisture content, their breakdown voltages for channel creation are different. Electrical discharges under lightning impulse conditions are propagated in small air pockets in the soil, the breakdown can happen repeatedly, usually without changing the characteristics of the soil [21].

If no other conditions are changed, then the higher the moisture content of soil, the shorter is the time to reach breakdown. The V-t curves show a typical pattern for breakdown characteristics of insulators. As moisture content levels increase, the amplitude of breakdown voltages decreases and the time-lag to breakdown becomes shorter. Electrical breakdown of the soil is initiated by ionization of the air trapped in the voids of the soil. The ionization gradient of the soil is always less than that of air and it is

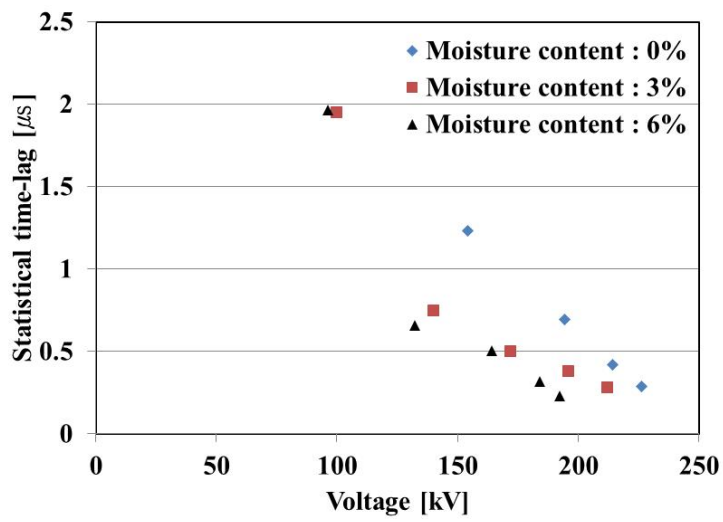

(a) Sand

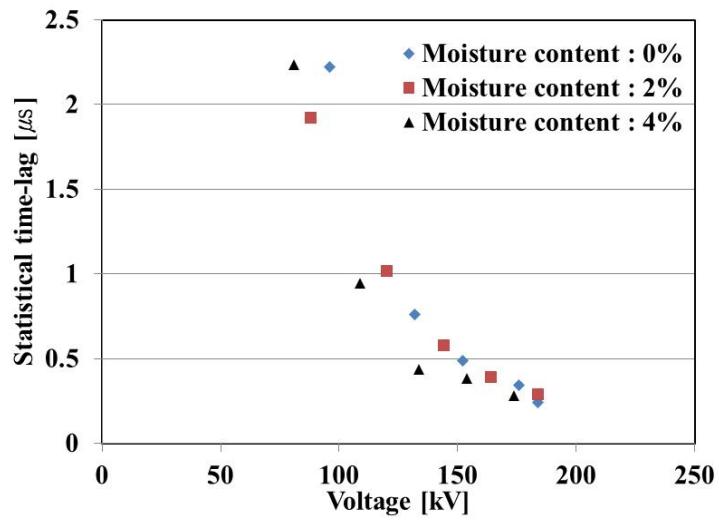

(b) Gravel

Fig. 8. Statistical time-lags as a function of moisture content in soils reduced when the moisture content is high [16]. The time to initiation of ionization is mainly dependent on the amplitude of impulse voltage, the moisture content, and the type and grain size of soil.

The statistical time-lag is the time which elapses during the voltage application until an ionization streamer appears to initiate the discharge. The statistical time-lags were plotted versus the peak of applied voltage in Fig. 8 for three moisture contents. The time elapsed from the application of impulse voltage to the initiation of ionization streamer, as defined and evaluated in this paper, represents the statistical time-lag in gas gaps.

The threshold level of the ionization is especially high when the test soil is dry or when it has a low conductivity. Also the water coating provides interconnected water paths which provide the low conductivity of the soil. The V-t curves will depend on the size of air voids between soil particles. The sand with fine particles will have smaller size voids, while the gravel with coarse particles will have larger size voids. The V-t curves for gravel is less irregular because the voids in gravel have less interfacial effect compared to those in sand.

The time-lag between the application of impulse voltage and the occurrence of breakdown is primarily dependent on

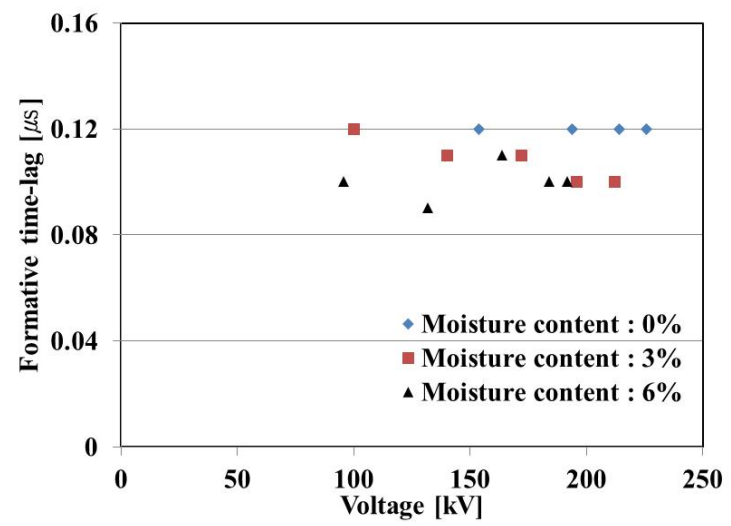

(a) Sand

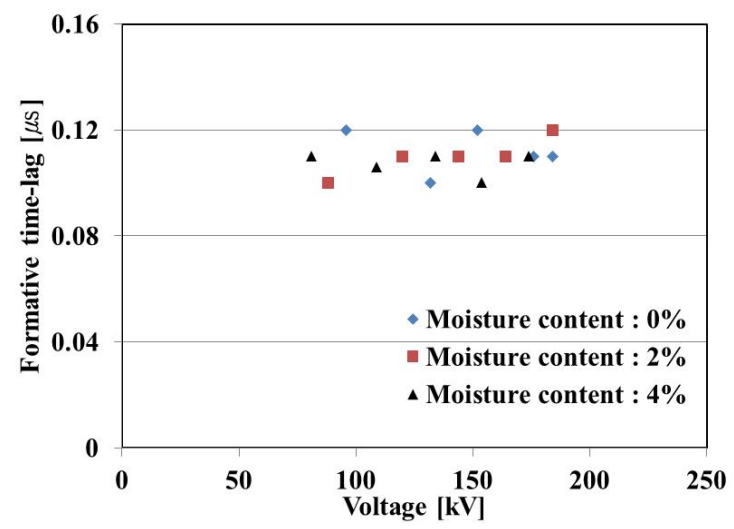

(b) Gravel

Fig. 9. Formative time-lags as a function of moisture content in soils 
the magnitude of applied voltages. The conduction current during the time from the application of impulse voltage prior to the initiation of ionization streamer is dominant and depends on the moisture content in soils. The time to initiation of ionization streamer is found to be shorter in soils with higher moisture content and decreases as the amplitude of applied voltage impulse increases. The dependency of the time to initiation of ionization streamer on the moisture content in sand is more important than that in gravel because the air pockets between sand grains are smaller than those found in the gravel soil.

Fig. 9 illustrates the formative time-lag to breakdown for sand and gravel as functions of moisture content. The formative time-lag, denoted $t_{2}$, is defined by the time interval from the initiation of ionization streamer to the breakdown. It corresponds to the time of discharge development and is closely related to the mechanism of discharge development.

According to Fig. 9, the formative time-lag is affected by the amplitude of applied voltage and the moisture content of soil. When the amplitude of applied voltage exceeds a certain value, the formative time-lags for different moisture content levels are similar. This statement is better seen for the gravel. The formative time-lags are slightly sensitive to the amplitude of impulse voltage. Their values are within the range of $0.09-0.12 \mu \mathrm{s}$. However, the variance of formative-time lags values is higher in sand than in gravel.

The electrical breakdown process in soil principally appears to be due to air ionization in the voids between soil particles [18]. It is inferred from the formative time-lags that the final jump of electrical breakdown is occurred by an ionization streamer. The moisture content of soil is the critical factor that affects the formation of breakdown channels. Soil breakdowns occur around the circumference of the top electrode. When the amplitude of applied impulse voltage exceeds a specified level, the soil is punctured by the tracking. The hole punched at the edge of top electrode is made by the electrical breakdown as shown in Fig. 10, and it indicates the observed effects of discrete breakdowns. However the electrode surface did not produce visible change. When the application of

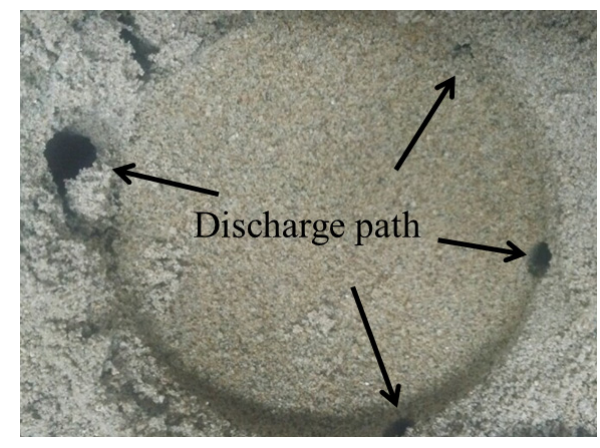

Fig. 10. Photograph of soil discharge impacts impulse voltage to the test soil is iterated, a satisfying repeatability is obtained. Electrical discharge would start at the edge of top electrode, where the current density is highest. It is obvious that the current is dispersed into the soil through a small part of the electrode surface. The electrical breakdown propagates with ionization caused by a combination of runs and pre-breakdown around the soil grains. The runs grow along irregular voids between the soil grains. The leading branch channel will experience the final jump bridging the soil between the two electrodes [22].

The discharge impacts made by the electrical breakdown are very resistive to the conduction current due to the evaporation around the breakdown channels and the formation of air pockets. The previous breakdown channel through the sample soil is obviously not a preferred path for a subsequent breakdown development, and the ionization streamers propagate in different paths from shot to shot [20]. Consequently, the electrical breakdown does not propagate along pre-formed channels in soils. When the impulse voltage is applied repeatedly, the electrical breakdown takes place along different branch paths, leading radially away from the stressed electrode. Hence, if the soil is left undisturbed between the applications of impulse voltages, subsequent breakdown in the soil is more likely to be occurred along a discrete channel than in previous breakdown channels.

\section{Conclusion}

In order to obtain valuable information about the effect of electrical discharges on the transient performance of grounding systems, we have investigated in this paper the physical and electrical phenomena of soil discharges as a function of moisture content when $1.2 / 50 \mu$ s lightning impulse voltage is applied. The results of this study can be summarized as follows:

(1) The electrical breakdown in soils is developed through two mechanisms: conduction current process and ionization process. The electrical breakdown is caused by ionization growths that follow the conduction currents.

(2) The time to breakdown is inversely proportional to the amplitude of lightning impulse voltage. The characteristics of the time-lag to the initiation of ionization streamer, which depend on the amplitude of applied voltage, are found to be similar to the $\mathrm{V}-\mathrm{t}$ curves.

(3) The time to initiation of ionization in soils is highly dependent on the amplitude of lightning impulse voltage, but the change in the formative time-lags with respect to the amplitude of applied voltage is negligible.

(4) When the lightning impulse voltage is continuously applied to the test soil, the electrical breakdown does 
not occur along previous channels, but makes a different discharge path other than punched holes.

All the obtained results in this paper present important guidelines to understand the transient behaviour of grounding systems under the passage of lightning currents

\section{Acknowledgments}

This work was supported by the New \& Renewable Energy Core Technology Program of the Korea Institute of Energy Technology Evaluation and Planning (KETEP), granted financial resource from the Ministry of Trade, Industry \& Energy, Republic of Korea. (No. No. 20133010011770)

\section{References}

[1] V. A. J. van Lint, J. W. Erler, "Electrical Breakdown of Earth in Coaxial Geometry", IEEE Trans. on Nuclear Science(NS), Vol. NS-29, No. 6, pp. 18911896, 1982.

[2] I. F. Genos, I.A. Stathopulos, "Soil ionization under lightning impulse voltages," IEE Proc.-Sci. Meas. Technol., Vol. 151, pp. 343-346, 2004.

[3] P. Espel, R. R. Diaz, A. Bonamy, and J. N. Silva, "Electrical Parameters Associated With Discharges in Resistive Soils," IEEE Trans. PD, Vol. 19, No. 3, pp. 1174-1182, 2004.

[4] N. Mohamad Nor and A. Ramli "Effects of Moisture content, impulse polarity and earth electrode's dimension on dry and wet sand under high voltage conditions", Euro. Trans. Electr. Power, John Wiley \& Sons, Ltd, DOI; 10.1002, 2007.

[5] L. Grcev, "Impulse Efficiency of Ground Electrodes", IEEE Trans. on PD, Vol.24, No.1, pp.441-451, 2009.

[6] Y. W. Yoo, S. C. Cho and B. H. Lee, "Ionization Characteristics of a Ground Rod with Radial Needle under Lightning Impulse", Proc. 5th ALPF, pp. 187190, 2008.

[7] S. Sekioka, M. I. Lorentzou, M. P. Philippakou, and J. M. Prousalidis, "Current-Dependent Grounding Resistance Model Based on Energy Balance of Soil Ionization", IEEE Trans. PD, Vol. 21, No. 1, pp. 194201, 2006

[8] Z. Song, M. R. Raghuveer and J. He, "Model for prediction of characteristics of lightning breakdown channels in soil in the presence of a buried cable", IEE Proc.-Genere. Transm. Distrib., Vol. 150, No. 5, pp. 623-628, Sep. 2003.

[9] N. Mohamad Nor, A. Haddad, and H. Griffiths, "Characterization of Ionization Phenomena in Soils under Fast Impulses”, IEEE Trans. PD, Vol. 21, No. 1, pp. 353-361, 2006.
[10] N. Mohamad Nor, A. Haddad, and H. Griffiths, "Performance of Earthing Systems of Low Resistivity Soils", IEEE Trans. PD, Vol. 21, No. 4, pp. 2039-2047, 2006.

[11] N. Mohamad Nor and A. Ramli, "Electrical properties of dry soil under high impulse currents", Journal of Electrostatics, Vol. 65, pp. 500-505, 2007.

[12] J. Wang, A. C. Liew, M. Darveniza, "Extension of Dynamic Model of Impulse Behavior of Concentrated Grounds at High Currents", IEEE Trans. on Power Delivery(PD), Vol. 30, No. 3, pp. 2160-2165, 2005.

[13] V. A. Rakov, et al, "New insights into lightning processes gained from triggered-lightning experiments in Florida and Alabama”, J. Geophys., Res., Vol. 103, No. D12, pp. 14, 117-14,130, 1998.

[14] Y. W. Yoo, "Analysis of Transient Ground Impedances Caused by Soil Discharges", Ph. D. dissertation, Inha University, Korea, pp. 2-3, 2015.

[15] IEEE Std 80-2000, IEEE Guide for Safety in AC Substation Grounding, IEEE Inc, pp. 49-63, 2000.

[16] Abdul M. Mousa, "The Soil Ionization Gradient associated with discharge of high currents into concentrated electrode", IEEE Trans, Vol. 9, No. 3, pp. 1669-1677, 1994.

[17] E. Kuffel, W. S. Xaengl and J. Kuffel, High Voltage Engineering - Fundamentals, 2nd ed., Butterworthheinemann, pp. 359 365, 2000.

[18] T. M. Flanagan, C. E. Mallon, R. Denson, and R. E. Leadon, "Electrical Breakdown Properties of Soil", IEEE Trans. on NS, Vol. NS-28, No. 6, pp. 44324439, 1981.

[19] IEC 60060-1, High-voltage test techniques - Part 1 : General definition and test requirements, pp. 14 22, 2001.

[20] J. W. Erler and D. P. Snowden, "High Resolution Studies of the Electrical Breakdown of Soil", IEEE Trans. on NS, Vol. NS-30, No.6, pp.4564-4567, 1983.

[21] A. Phillips, Guide for Transmission Line Grounding, A Roadmap for Design, Testing, and Remediation: Part 1 - Theory Book, EPRI, pp. 5-47 5-52, 2006.

[22] B. H. Lee, G. H. Park, H. G. Kim, and K. S. Lee, "Analysis of Soil Ionization Behavior under Impulse Currents", J. Electrical Engineering \& Technology, Vol. 4, pp. 99-105, 2009.

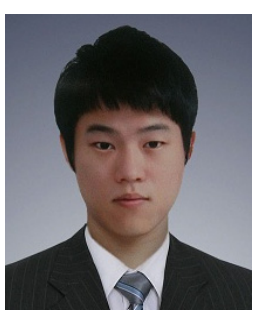

Seung-Min kim He received his B.S. degree in Physics from Inha University in 2011 and is pursuing his M.S. degree at the same University. His research interests are in the area of surge protection and grounding systems. 


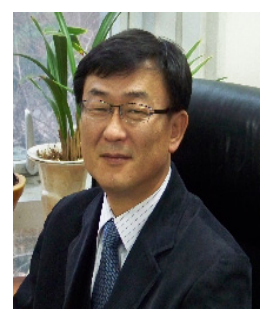

Yang-Woo Yoo He received his Ph.D. degree in Electrical Engineering from Inha University in 2015. He worked in the areas of R\&D management of Korea Electrical Engineering \& Science Research Institute since 1993. He has been working at KwangMyung Electric co., Ltd as a senior managing director from 2012. His branch research is lightning protection and grounding system.

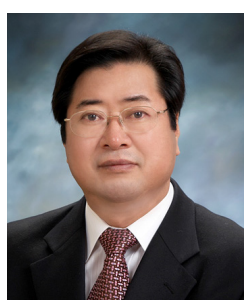

Bok-Hee Lee He received his Ph.D. degree in Electrical Engineering from Inha University in 1987. He has been with the school of Electrical Engineering at Inha University, Inchon, Korea, as an Assistant Professor since 1990, becoming a Professor in 1999. From 1988 to 1989 , he was a postdoctoral research fellow at the Institute of Industrial Science, University of Tokyo. From Apr. 1999 to Feb. 2000, he was a Visiting Professor in the University of Cincinnati. His research interests are in the area of lightning, lightning protection, grounding systems, surge protection, electrical discharges, high voltage engineering and electromagnetic compatibility. 